\title{
Current Trends and Research Challenges in Spectrum- Sensing for Cognitive Radios
}

\author{
Roopali Garg*, \\ UIET, Panjab University, \\ Chandigarh, India
}

\author{
Dr. Nitin Saluja \\ CURIN, Chitkara University, \\ Rajpura, Punjab, India
}

\begin{abstract}
The ever increasing demand of wireless communication systems has led to search of suitable spectrum bands for transmission of data. The research in the past has revealed that radio spectrum is under-utilized in most of the scenarios. This prompted the scientist to seek a solution to utilize the spectrum efficiently. Cognitive Radios provided an answer to the problem by sensing the idle (licensed) bands and allowing (secondary) users to transmit in these idle spaces. Spectrum sensing forms the main block of cognition cycle.

This paper reviews the current trends in research in the domain of spectrum sensing. The author describes the type of channel being modelled, diversity combining schemes used, optimal algorithms applied at fusion centre, spectrum sensing techniques employed. Further, the research challenges are discussed. It is presented that various attributes like sensing time, throughput, rate reliability, optimum cooperative users, sensing frequency etc. needs to be addressed. A trade-off needs to be established to optimize two opposing parameters like sensing and throughput.
\end{abstract}

Keywords-CR; cognitive radio; FC-PSO; fast-convergence particle swarm optimization; FC; fusion centre; KLMS; kernel least mean square; $P U$; primary use); ROC; receiver operating characteristic curves; $S U$; secondary user; soft combination; spectrum hole

\section{INTRODUCTION}

The scarcity of available spectrum and the inefficient usage of the same motivated the researchers to look for solutions in Dynamic Spectrum Access (DSA). DSA networking was introduced by Defense Advanced Research Projects Agency (DARPA) which caused a paradigm shift from traditional fixed spectrum access to dynamic spectrum access. [1] [2] [3].

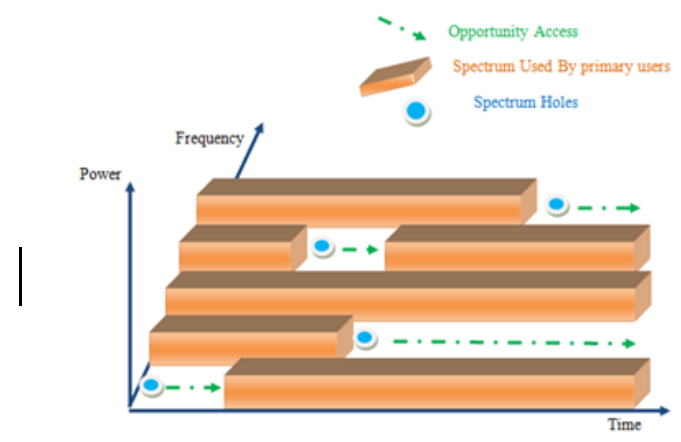

Fig. 1. Spectrum Hole
Cognitive Radio (CR) is the enabling technology for dynamic spectrum access. The idea of cognitive radio was first conceived by Joseph Mitola III. The latin word 'Cognoscere' forms the root of term 'Cognition'. It means 'to know' [4]. CRs have the capability to sense the radio environment. They can choose the portion of spectrum that is unused. These portions are referred to as Spectrum holes or white spaces (as shown in figure 1). Thereafter, the data can be transmitted on this chosen band without interference with the licensed user.

Primary Users (PU) are users having access rights to licensed spectrum band. On the other hand, Secondary Users (SU), have cognitive radio capabilities. They have the ability to sense the surroundings for availability of unused band. They request the PU to make use of this unused spectrum for wireless communication. PU have higher priority over SU. SUs ensures that they do not cause interference to PUs.

Cognition cycle involves the function of sensing the spectrum, making a decision about the hole and the licensed user, sharing the spectrum and mobility of secondary user in case a licensed user is detected. Accordingly, the four phases of cognition cycle can be described as: Spectrum Sensing is one of an important building block of cognitive radio. It involves the task of sensing the radio environment for the presence of spectrum holes and detection of PUs. Spectrum decision decides for the optimum selection of spectrum hole to transmit the data. As there are several CR users sharing the same spectrum, there is a need for a mechanism which coordinates the network access to all specified users. This can be defined under Spectrum Sharing. Under Spectrum Mobility if any primary licensed user is detected, then the CR should seamlessly switch over to some other suitable spectrum hole for further transmission [5]. Spectrum sensing encounters the issues like fading, shadowing and noise uncertainty. The scheme of cooperation has been suggested by researchers as an answer to these problems [6]. Here, CR users cooperate to share their sensing information for making a combined decision which is usually more accurate than individual decision. It reduces the probability of false alarm and misdetection. Moreover, it solves the hidden primary user problem and reduces the sensing time [7].

The raw or processed data from each user is sent to a data fusion centre. It processes this collected data and finally makes a decision. The implementation of Cooperative sensing can be classified as Centralized Sensing [8], Distributed Sensing [9] , External Sensing and Relay Assisted Sensing [6] depending on presence of fusion centre or use of multi-hop for sensing. 
The rest of the paper is divided into six sections. The hypothesis governing the absence or presence of licensed user is presented in Section II. Section III highlights the techniques employed for carrying spectrum sensing. Section IV presents the latest trends in the domain of study. The research challenges are described in Section V. Finally the paper is concluded in section VI and is appended with references.

\section{PROBLEM FORMULATION}

The frame structure of a CR (as depicted in figure 2) consists of sensing time $T_{s}$ followed by data transmission time $T_{t}[10]$ i.e $T_{s}+T_{t}=T_{f}$; Where $T_{f}$ is the frame period and the sensing frequency is $1 / \mathrm{T}_{\mathrm{s}}$.

Spectrum sensing can be formulated with two hypotheses :

$H_{0}$ : Channel is vacant temporarily i.e PU is absent

$H_{1}$ : Channel is occupied i.e PU is present

Thus the spectrum sensing problem is to decide between

Null Hypothesis $H_{0}$ :

$$
Y[n]=U[n] \quad: P U \text { is absent }
$$

Alternate Hypothesis $H_{l}$ :

$$
Y[n]=h . X[n]+U[n] \quad: P U \text { is present }
$$

Here $n=1,2,3 \ldots \ldots \mathrm{N}$, where $\mathrm{N}$ is the number of samples and $h$ is the channel gain. It is considered equal to 0 under null hypothesis $\mathrm{H}_{0}$ and 1 under alternate hypothesis $\mathrm{H}_{1}$.

$\mathrm{Y}[\mathrm{n}]$ is sensed signal by $\mathrm{SU} . \mathrm{X}[\mathrm{n}]$ represents the primary signal. It is assumed to be i.i.d (independent and identically distributed) random process with mean zero and variance $E\left[|X(n)|^{2}\right]=\sigma_{x}{ }^{2}$. $\mathrm{U}[\mathrm{n}]$ represents noise added by the wireless channel. It is assumed to be Gaussian and i.i.d random process with mean zero and variance $E\left[|U(n)|^{2}\right]=$ $\sigma_{u}^{2}$

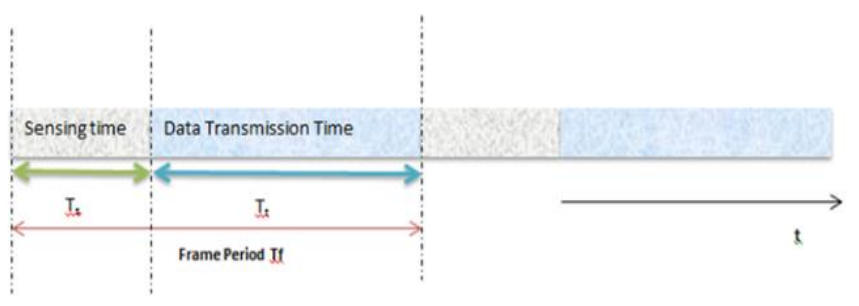

Fig. 2. Frame Structure of Cognitive Radio

A threshold is defined as per the specification of grade of services. If test value of $\mathrm{Y}[\mathrm{n}]$ is greater than threshold then, alternate hypothesis $\mathrm{H}_{1}$ is assumed to be accepted else null hypothesis $\mathrm{H}_{0}$ is accepted.

Sensing accuracy is determined by Receiver Operating Characteristic (ROC) curves. These curves are the plot of probability of false alarm with probability of accurate detection or plot of probability of miss detection with probability of false alarm. These probabilities can be formulated as under:

Probability of detection: It is the probability that CR user will declare the presence of PU truly when it is present. A miss in detection of PU will lead to interference with the PU.

$$
P_{d}=P_{r}\left\{\text { Decision } H_{1} \mid H_{1}\right\}
$$

Probability of false alarm: is probability that CR user will declare the presence of Primary user when it is actually not present.

$$
P_{f}=P_{r}\left\{\text { Decision } H_{1} \mid H_{0}\right\}
$$

Probability of miss detection: Probability of missing the signal when it was actually present.

$$
P_{m}=P_{r}\left\{\text { Decision } H_{0} \mid H_{1}\right\}
$$

Probability of accurate detection: It is sum of probability of detection if PU is present and probability of no detection as $\mathrm{PU}$ is absent.

$$
P_{a d}=P_{r}\left\{\text { Decision } H_{1} \mid H_{1}\right\}+P_{r}\left\{\text { Decision } H_{0} \mid H_{0}\right\}
$$

\section{SPECTRUM SENSING TECHNIQUES}

The main objective of sensing methods is to detect the spectrum holes so that the SU can use these vacant bands. The methods for spectrum sensing are classified in figure 3 and the description of each is given in the following sub-section.

\section{A. Prior Information Needing}

1) Matched Filtering ( $M F)$

In this methodology, the concept of matched filter detection technique is applied. Here an unknown signal $\mathrm{x}(\mathrm{t})$ is convolved with filter impulse signal $h(t)$.Prior knowledge of bandwidth requirement, operating frequency, frame format, pulse shaping and modulation types etc is needed [11]. The advantage offered by Matched filtering technique is that it offers high probability of detection in less sensing time. It is considered as the best method in this category. Even with less signal samples detection is good. It is robust to noise uncertainty. Moreover, it offers good detection even at low SNR. On the other hand, the implementation is quite complex and involves large power consumption. In addition, there is a need for precise information about certain waveform patterns of PU [12].

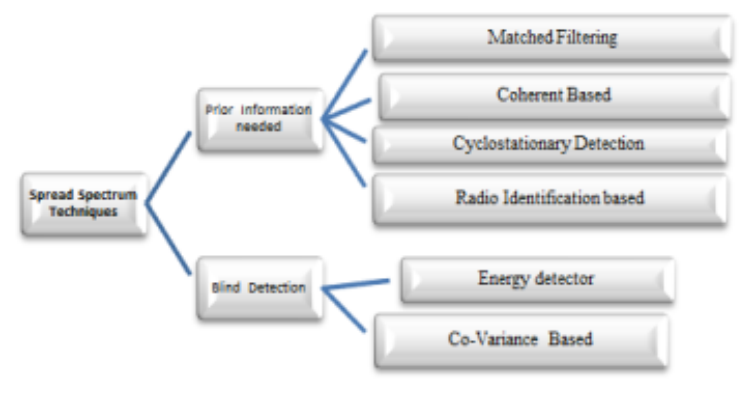

Fig. 3. Classification of Spectrum Sensing Techniques

\section{2) Cyclostationary Detection (CSD)}

This method utilizes the cyclostationary features like operating frequency, required bandwidth, frame format and modulation types from received signal of PU statistics like mean, cyclic correlation and autocorrelation. Periodicity in the signal causes cyclostationary features [13].

The robustness to uncertainty in noise power and propagation channel makes it attractive. However, there is need for high sampling rate. As large number of samples are 
needed, it adds to the complexity. Also, the sensing time is high [14].

\section{3) Coherent detection}

In coherent detection, preambles, mid-ambles and pilot patterns are regularly transmitted. A preamble is a sequence (known already) which is sent before each slot. The sequence which is transmitted in the middle of the slot is known as midamble. When the information of these known patterns is available, received signal is correlated with its own known copy. Thus it assists in signal detection [10].

When there is long known primary signal pattern, the accuracy gets better. The stumbling block of this method is that huge amount of PU information is needed for the signal patterns to witness high performance. But it is not practically possible to achieve such large amount of information. Coherent detection technique is more reliable and has less convergence time than energy detector technique [15].

\section{4) Radio Identification Based Detection}

The main concept used in this technique is to identify the presence of some known technology and to achieve communication using this technology. If the technology used by primary user for transmission is known, then good knowledge can be derived about the spectrum characteristics. In addition it imparts higher accuracy. Eg: if the primary user is using bluetooth, then the $\mathrm{CR}$ can use this information to gain some knowledge in space dimension. Here, it uses the knowledge that bluetooth operates in the range of $10 \mathrm{~m}$. Then CR device may use bluetooth to communicate for some applications in the range supported by bluetooth. The detection process is highly accurate, has average sensing time. Also, it is robust to SNR. Never-the-less, high power consumption due high complexity makes it unattractive [16].

\section{B. Blind Detection}

\section{1) Energy Detector}

Energy detector is the most commonly used method commercially. The decision is made by comparing the output of signal detector with a fixed threshold value which helps in deciding the presence or absence of PU [16]. The attractive features of this methodology are that it is simple and easy to implement. It requires less sensing time and has low power consumption. Nonetheless, the noise uncertainty leads to increase in probability of false alarm. It is very unreliable in low SNR regime. Also, it is unable to distinguish between PU from other signal source. This technique has low accuracy as compared to other techniques [17] [18]. An energy detector is ineffective in detecting spread spectrum signals [19].

\section{2) Co-variance Based Sensing}

The spectrum sensing using co-variance technique works on the concept of comparison between covariance of the detected signal with the covariance of noise [20].The probability to differentiate between signal and noise is too high even at low values of SNR. The power consumed is low though increased complexity, increased computational overhead are the weakness of this methodology. Moreover, it has low detection performance and proves inefficient in case of spread spectrum signal [21].

\section{CURRENT TRENDS}

The current trends in spectrum sensing jointly deals with several attributes, addresses a trade-off between this parameters and suggests algorithms to converge to an optimal solution.

Rashid in [22] proposed a fast-convergence particle swarm optimization (FC-PSO) scheme. It is fine sensing scheme to find a trade-off between sensing time and throughput. The parameters considered in the study are detection performance, optimization time, and SU gain. The paper utilized energy detection scheme for in-band spectrum sensing. Detection performance, optimization time, and SU gain are some of the key parameters considered in this paper. A trade-off problem was formulated between the stopping threshold, sensing performance and the optimization time. Traditionally, the stopping criterion was based on measurement of error or limiting the maximum number of iterations. However, FCPCO algorithm imposed a limit rule to stop the evaluation. When a certain number of particles reach a global optimum, the algorithm stopped. This is based on the fact that the remaining particles will not produce any new information and will be a waste of computational resources.

Additionally, the proposed algorithm was compared with Exhaustive Search Algorithm, Golden Section Search (GSS) algorithm, Artificial Immune System (AIS) algorithm, PSO algorithm. It was concluded that proposed FC-PSO algorithm has fastest convergence time, better computational complexity and was energy efficient.

However, as the frame duration is kept constant in this research, the future direction steer towards the study of frame optimization. Additionally, the factors affecting the choice of threshold, is unknown. So, methodologies can be devised to search for the best threshold value. Further, in order to detect a PU using cooperative spectrum sensing, a joint decision of an optimum number of users can be taken.

Serkan in [23] proposed a novel spectrum sensing scheme of SU and PU users without any prior knowledge. Here, a known amount of noise is intentionally added at the sensing side. Thus the sensing detector becomes unaffected to signal and noise types, thereby diminishing the problem of noise uncertainty. As this additional noise is removed after the sensing process, there is no effect on the transmission process. This scheme has been found to be robust under Rician and Nakagami fading models. The performance analysis of the algorithm was carried on real data and the experimental results showed probability of detection to be 0.90 for SNR values of $10 \mathrm{~dB}$.

Xiguang in [24] utilizes Kernel Least Mean Square (KLMS) algorithm for proposing a novel cooperative spectrum sensing scheme. Each SU uses energy detection technique to take a binary decision for spectrum sensing. This decision, based on KLMS algorithm, is sent to fusion center to make the final decision on the status of occupancy of the spectrum. The proposed technique can keep track of changing environment and increase the reliability of decisions in FC considerably because KLMS performs very well in judging a complex non-linear mapping in an online manner. The Monte- 
Carlo simulations confirm the desirable performance of this innovative scheme.

In this paper, ROC curves are presented showing the comparison of proposed scheme with some of the existing schemes like Energy Detector, Cooperative Energy Detection, Anderson-Darling based detection and Kolmogorov-Smirnov based detection. It was shown that the new technique provided a maximum probability detection of 0.984 as compared to the above mentioned techniques for probability of false alarm of 0.1 and average SNR of $-8 \mathrm{~dB}$.

Bagheri in [25] has derived analytical expressions for Maximal Ratio Combining (MRC) and Square Law Combining (SLC) schemes. Each secondary user senses the spectrum with Energy Detectors. The channels were modelled as Nakagami-m multipath fading and lognormal shadowing. K-out-of-n fusion decision rule was used. The Least Mean Square (LMS) algorithm was utilized at the fusion centre to detect presence or absence of PU.

Xin-Lin in [26] derived a mathematical model between spectrum sensing frequency and number of remaining packets that need to be sent; spectrum sensing frequency and the new channel availability time during which the cognitive radio networks is allowed to use a new channel (after the current channel is re-occupied by primary users) to continue to transmit the packet. Spectrum sensing frequency is how frequently a CR user detects the free spectrum.

Higher spectrum sensing frequency increases Media Access Control (MAC) layer processing overhead and delay. This can cause some multimedia packets to miss the receiving deadline. Thus the multimedia quality at the receiver side is decreased. A smaller number of remaining packets and a larger value of new channel availability time will help to transmit multimedia packets within a delay deadline. HughesHartogs and DPSO algorithms are used to obtain the optimal solution in multi-channel case.

Herath in [27] studied the performance of the energy detector with diversity reception. The fading Channels used in the study were Nakagami-m and Rician fading channel. Maximal Ratio Combining (MRC), Equal Gain Combining (EGC) and Selection Combining (SC) diversity schemes were utilized. For the EGC diversity case, with Nakagami-m fading, $P_{d}$ expressions are derived for the cases $L=2,3,4$ and $L>4$. For the SC diversity case, with Nakagami-m fading, $P_{d}$ expressions are derived for the cases when $L>=2$. Here, $L$ is number of diversity branches.

Stotas in [28] proposed a novel method to address the sensing-throughput problem. Here, both sensing time and throughput were maximized. Spectrum sensing and transmission of data was done at the same time for whole frame duration. A time slot was allocated for spectrum sensing at the beginning of each frame. During this slot, data transmission was prohibited. This resulted in less false alarms and better probability of detection. Energy detectors were employed for spectrum sensing. The frame duration was fixed at $100 \mathrm{~ms}$. The received SNR from SU was kept at $20 \mathrm{~dB}$. The bandwidth of the channel and the sampling frequency was chosen as $6 \mathrm{MHz}$. The probability that the frequency band is active was taken as 0.2 .

Tevfik in [10] presented a survey and comparison of various techniques used for spectrum sensing such as energy detector based, waveform based, cyclostationary based, radio identification based and matched filtering based. It describes various aspects of spectrum sensing for cognitive radio. The concept of sensing in multi-dimension like frequency, time, space, code and angle was also introduced. Further, the various challenges associated with spectrum sensing were studied. Additionally, the fundamental behind cooperative sensing and its several types was explained

Liang in [29] mathematically formulated a sensingthroughput problem and validated the same with simulations. It considered the issue of modelling the sensing time while maximizing the throughput for the SU under the constraint that the interest of PUs was sufficiently guarded. The sensing scheme used for the same was energy detection. The frame duration was fixed at $100 \mathrm{~ms}$, SNR of PU at secondary receiver was taken as $-20 \mathrm{~dB}$ with $6 \mathrm{Mhz}$ Channel and $\mathrm{P}_{\mathrm{d}}=0.9$. It was researched that optimal sensing time that gave highest throughput was $14.2 \mathrm{~ms}$. On employing distributed spectrum sensing the optimal sensing time reduced to $9.5 \mathrm{~ms}$. For distributed spectrum sensing, 4 distributed SUs were utilized which worked cooperatively to carry out sensing using LogicAND decision fusion rule.

Kim in [18] proposed an optimal in-band sensing scheduling algorithm. The sensing-time and sensing-frequency of energy and feature detection were optimized. The factors taken into consideration were noise uncertainty and inter-CRN interference. It was observed that energy detection above average Received Signal Strength ( $\operatorname{aRSS}_{\text {threshold }}$ ) incurred at most $0.385 \%$ of sensing overhead. This overhead was compared with three feature detectors: pilot-location detection, PN511 detection, cyclostationary detection. In this paper, aRSS threshold $_{\text {was }}$ varied from $-114.6 \mathrm{dBm}$ to -109.9 $\mathrm{dBm}$. The noise uncertainty values were kept between $0.5 \mathrm{~dB}$ to $2 \mathrm{~dB}$ and $-112.9 \mathrm{dBm}$ to $-110.5 \mathrm{dBm}$. The interfering Cognitive Radio Networks were in the range of 1 to 6 .

\section{RESEARCH CHALLENGES}

There are several factors inspiring the scientists across the globe to study the problems faced during sensing. These factors need to be addressed jointly to converge to an optimum solution.

- The information regarding the presence of PU is mostly missing in commercialized applications of wireless communication systems. Another hurdle is the inability to distinguish between PU and the noise. Therefore the need for blind algorithms to sense the PU is obvious as the algorithms perform without prior information about the channel or primary signals.

- As the SNR values at the sensing detector side can be as low as $-1 \mathrm{~dB}$, hence the need is to have the detectors which are agile enough to detect under low SNR values. 
- Depending on the type of application, the communication channel is prone to several types of noises. At the sensing detector, there is always a noise uncertainty. Thus detection process should be robust with constraint of noise uncertainty.

- The sensing time should be as small as possible so as to not compromise with the throughput. Thus the need is to have fast and less complex sensing operations.

- The selection of optimum threshold values for spectrum sensing techniques like Energy detection is critical because this forms the basis for decision for absence or presence of PU.

- Various types of feature detectors can be compared with the energy detectors.

- Delay analysis in distributed schemes: Distributed cooperative sensing schemes utilizes a repetitive procedure to make a final cooperative decision. This leads to cooperative sensing delay. If to converge to a decision, a large number of iteration is needed, then report delays would be large. Thus, delay analysis and the time taken to converge to a decision needs to be jointly examined [6] [30].

- Cooperation-Processing Trade-Off: If there are large number of cooperative users, probability of detection increase even if there are low-sensitive detectors possessed by each user. A detector with less sensitivity requirement leads to shorter sensing time and therefore less local processing.

However, large number of cooperative users causes large overhead as there is large volume of data that needs to be reported and centrally processed. This increased overhead causes an increase in the sensing time and the processing of data. Thus there is a need for a trade-off between them.

- Reactive Vs. Proactive Sensing: In reactive sensing scheme, the spectrum sensing is performed only when data needs to be transmitted. In contrast, proactive schemes maintain a list of idle licensed bands available for opportunistic access. The later scheme helps in reduction of sensing time. However, both the mentioned spectrum sensing techniques involve high sensing overhead. The reduction of sensing overhead thus is always a challenge. The applications which are very sensitive to delays may find proactive sensing attractive. For instance, while searching an overcrowded band with few spectrum holes may increase delay considerably if on-demand scheme is followed. While, the applications having energy efficiency constraints but are delay tolerant may prefer reactive sensing. Thus a CR needs to adapt to either of the sensing mode (reactive or proactive) in order to achieve optimum performance. The implementation of such system requires parameters to be optimized [31].

- Rate-Reliability Trade-Off: Whenever a PU is detected, the CR user needs to stop transmission and relocate to a new idle band. Though the delays associated with these relocations may be reduced using proactive scheme, yet cognitive users have to bear Quality of Service (QoS) degradation. This is due to the fact that communication peers have to coordinate for the frequency transition. The communication reliability can be increased by spreading the transmission data over wide spectrum. So that, if a PU reclaims this band, it affects only a small portion of cognitive user's bandwidth. At the receiver side, the data from several frequency chunks can be combined to form a reliable CR link. Orthogonal-Frequency-Division-Multiplexing (OFDM) can be utilized for this purpose due to its inherent flexibility in using non-adjacent frequency bands. The challenge posed by this method is that additional temporal or spectral resources have to be allocated for periodic sensing of these extra frequency bands which reduces the effective data rate of CR user [30].

- Sensing-Throughput: If the sensing time is increased, it increases the probability of detection $\mathbf{P}_{\mathbf{d}}$ and decreases the probability of false alarm $\mathbf{P}_{\mathbf{f}}$. However this results in decreased transmission time $\mathbf{T}_{\mathbf{t}}$. This results in reduced throughput of $\mathrm{CR}$. Thus, sensing time and throughput needs to be optimized [22] [29].

- Reporting Time - Sensing Time: Jaewoo in [32] formulated a problem to maximize the average capacity of SU by jointly taking into account the sensing time, reporting time, a fusion scheme and soft combination at fusion centre. Also, a limited reporting scheme for a multiband cooperative spectrum sensing was proposed. This scheme proved useful in increasing the average SU capacity by reducing the reporting overhead while providing desirable protection to primary user's interest. Probability of false alarm was also calculated. It was inferred that with 40 cooperative users, the proposed scheme gave a rise of $21 \%$ in average capacity of SU as compared to conventional schemes.

\section{CONCLUSION}

Radio spectrum is scarce. Therefore there is a need to efficiently utilize it. Cognitive radios employ the concept of dynamic spectrum reuse. They intelligently identify the spectrum holes in licensed-bands which are used by primary users, and dynamically allow the secondary users to transmit in these spaces. The absence or presence of a licensed user is governed by null hypothesis or alternate hypothesis. The spectrum sensing techniques can sense with prior knowledge of signal and power or can use blind detection techniques. When a large number of cognitive users operate in cooperation, then the probability of false alarm reduces. Further, this provides answer to issues like hidden primary user problem, fading, shadowing and noise uncertainty. This article reviews the ongoing research in the domain of spectrum sensing. Here types of channel modelled, various spectrum sensing techniques employed, diversity combining schemes used, traditional and optimised algorithms at the fusion centre are discussed. 


\section{OPEN PROBLEM}

The future directions in this domain hold the need for optimization of techniques used in spectrum sensing and cooperative spectrum sensing. The attributes that can be investigated are probability of false alarm, probability of detection, SNR values, throughput, sensing time etc. The issue like selection of threshold value to detect the PU needs to be studied. The sensing time should be as small as possible but not at the cost of throughput. Moreover, sensing can be done on demand or proactive basis.

\section{ACKNOWLEDGMENTS}

The authors would like to thank Panjab University for allowing to conduct the research at University Institute of Engineering \& Technology. The study could not have been possible without the constant support of Dr. Ashok K. Chitkara, Chancellor, Chitkara University. Deep gratitude to Mr. Abhishek Pal Garg, MBA (INSEAD, France), Deputy Secretary, Government of India for proof reading and providing valuable feedback. Many thanks to the anonymous reviewers, whose insightful comments made this a better paper.

\section{REFERENCES}

[1] C. Xin, M. Song, L. Ma and C. C. Shen, "ROP: Near optimal rendezvous for dynamic spectrum access networks," IEEE Transactions on Vehicular Technology, vol. 62, no. 7, pp. 3383-3391, 2012.

[2] M. Song, C. Xin, Y. Zhao and X. Cheng, "Dynamic spectrum access: from cognitive radio to network radio," IEEE Wireless Communications, vol. 19, no. 1, pp. 23-29, 2012.

[3] B. Zhang, Y. Chen and K. Liu, "An indirect-reciprocity reputation game for cooperation in dynamic spectrum access networks," IEEE Transactions on Wireless Communication, vol. 11, no. 12, pp. 43284321, 2012.

[4] L. Giupponi, A. Galindo-Serrano, P. Blasco and M. Dohler, "Docitive networks: An emerging paradigm for dynamic spectrum management," IEEE Wireless Communication, vol. 17, no. 4, pp. 47-54, 2010.

[5] I. F. Akyildiz, W. Y. Lee, M. C. Vuran and S. Mohanty, "A survey on spectrum management in cognitive radio networks," IEEE Communication Magazine, vol. 46, no. 40, pp. 40-48, 2008.

[6] I. F. Akyildiz and R. Balakrishnan, "Cooperative spectrum sensing in cognitive radio networks: A survey," Physical Communication, vol. 4, no. 1, pp. 40-62, 2011.

[7] D. Cabric, A. Tkachenko and R. Brodersen, "Spectrum sensing measurement of pilot, energy and collaborative detection," in IEEE Military Communication Conference, Washington D.C, USA, 2006.

[8] L. Lu, X. Zhou, U. Onunkwo and G. Y. Li, "Ten years of research in spectrum sensing and sharing in cognitive radio," EURASIP Journal of Wireless Communications and Networkings, pp. 1-16, 2012.

[9] B. Wang and K. R. Liu, "Advances in cognitive radios: A survey," IEEE Journal of Selected Topics in Signal Processing, vol. 5, no. 1, pp. 5-23, 2011.

[10] T. Yücek and H. Arslan, "A survey of spectrum sensing algorithms for cognitive radio applications," IEEE Communications Surveys \& Tutorials, vol. 11, no. 1, pp. 116-130, 2009.

[11] S. Shobana, R. Saravanan and R. Muthaiah, "Matched filter based spectrum sensing on cognitive radio for OFDM WLANs," International Journal of Engineering and Technology, vol. 5, no. 1, pp. 142-146, 2013.

[12] N. Giweli, S. Shahrestani and H. Cheung, "Spectrum sensing in cognitive radio networks: QoS considerations," in Seventh International Conference on Networks \& Communications NETCOM 2015, Sydney, Australia, 2015.

[13] K. Kim, I. A. Akhbar, K. K. Bae, J. S. Um, C. M. Spooner and J. H. Reed, "cyclostationary approaches to signal detection and classification in cognitive radio," in Symposium on New Frontiers in Dynamic Spectrum Access Networks, Dublin, 2007.

[14] J. Lunden, A. Koivunen, A. Huttunen and H. V. Poor, "Spectrum sensing in cognitive radios on multiple cyclic frequencies," in $2 n d$ International Conference on Cognitive Radio Oriented Wireless Networks and Communications CrownCom-2007, Orlando, Florida, USA, 2007.

[15] H. Tang, "Some physical layer issues of wideband cognitive radio systems," in International Symposium on New Frontiers in Dynamic Spectrum Access Networks, Baltimore, USA, 2005.

[16] T. Yücek and H. Arslan, "Spectrum characterization for opportunistic cognitive radio systems," in IEEE Military Communication Conference, Washington D.C, USA, 2006.

[17] Y. Zeng, Y.C Liang, A.T. Hoang and R.Zhang;" "A review on spectrum sensing for cognitive radio challenges and solutions," EURASIP Journal of Advances in Signal Processing, vol. 10, 2010.

[18] H. Kim and K. G. Shin, "In-band spectrum sensing in cognitive radio networks: Energy detection or Feature detection?," in 14th ACM International Conference on Mobile Computing and Networking, San Fransico, California, USA, 2008.

[19] D. Cabric, S. M. Mishra and R. W. Brodersen, "Implementation issues in spectrum sensing for cognitive radios," in Thirthy-Eight Asilomar Conference on Signals, Systems and Computers, Pacific Grove, California, USA, 2004.

[20] Y. Zeng and Y. C. Liang, "Spectrum-sensing algorithms for cognitive radio based on statistical covariances," IEEE Transactions on Vehicular Technology, vol. 58, no. 4, pp. 1804-1815, 2009.

[21] D. B. Rawat and G. Yan, "Spectrum sensing methods for dynamic spectrum sharing in cognitive radio networks: A survey," International Journal of Research and Reviews in Wireless Sensor Networks, vol. 1, no. 1, pp. 1-13, 2011.

[22] R. A. Rashid, A. Rashid, A. H. B. A. Hamid, N. Fisal, S. K. Syed-Yusof and H. Hosseini, "Efficient in-band spectrum sensing using swarm intelligence for cognitive radio network," Canadian Journal of Electrical and Computer Engineering, vol. 38, no. 2, pp. 106-115, 2015.

[23] S. Ozbay and E. Ercelebi, "A new wireless network scheme for spectrum sensing in cognitive radio," Elektronika Ir Electrotechnika, vol. 21, no. 6, pp. 90-95, 2015.

[24] X. Xu, R. Qu, J. Zhao and B. Chen, "Cooperative spectrum sensing in cognitive radio networks with kernel least mean square," in 5th International Conference on Information Science and Technology (ICIST), China, 2015.

[25] A. Bagheri, A. Shahini and A. Shahzadi, "Analytical and learning-based spectrum sensing over channels with both fading and shadowing," in International Conference on Connected Vehicles and Expo, Nevada, USA, 2013.

[26] H. Xin-Lin, W. Gang, H. Fei and S. Kumar, "The impact of spectrum sensing frequency and packet-loading scheme on multimedia transmission over cognitive radio networks," IEEE Transactions on Multimedia, vol. 13, no. 4, pp. 748-761, 2011.

[27] S. P. Herath, N. Rajatheva and E. Tellambura, "Energy detection of unknown signals in fading and diversity reception," IEEE Transactions on Communications, vol. 59, no. 9, pp. 2443-2453, 2011.

[28] S. Stotas and A. Nallanathan, "Overcoming the sensing-throughput trade-off in cognitive radio network," in IEEE International conference on Communication (ICC), Cape Town, South Africa, 2010.

[29] Y. Liang, Y. Zeng, E. Peh and A. Hoang, "Sensing throughput tradeoff for cognitive radio networks," IEEE Transaction Wireless Communication, vol. 7, no. 4, pp. 1326-1337, 2008.

[30] A. Ghasemi, "Spectrum sensing in cognitive radio networks: Requirements, challenges and design trade-offs," IEEE Communications Magazine, vol. 46, no. 4, pp. 32-39, 2008.

[31] L. Wang and C. Wang, "Spectrum handoff for cognitive radio networks: Reactive-sensing or Proactive-sensing?," in IEEE International Conference on Performance, Computing and Communications, Austin, Texas, USA, 2008. 
[32] J. So and T. Kwon, "Limited reporting-based cooperative spectrum sensing for multiband cognitive radio networks," International Journal of Electronics and Communications, vol. 70, no. 5, pp. 1-12, 2016. 